\title{
The magnetic field of an ellipsoidal star
}

\begin{abstract}
V. R. Khalack
Main Astronomical Observatory, 27 Zabolotnogo Str., 03680, Kyiv, Ukraine

e-mail: khalack@mao.kiev.ua

Received 10 January 2004 / Accepted 6 August 2004

Abstract. The approximate expressions for the mean longitudinal magnetic field, crossover effect and quadratic magnetic field are deduced in the framework of the modified magnetic charge description method for an ellipsoidal star, that is represented by the oblique rotator model with the centered symmetric magnetic dipole. For the mean crossover effect and the mean quadratic magnetic field the effect of stellar oblation increases their theoretical values (obtained for the case of a spherically symmetric star) up to $12 \%$ and up to $8 \%$ respectively. This can lead to the essential overestimation of the magnetic dipole strength value obtained from the mean crossover effect and quadratic magnetic field observations if one does not pay attention to the ellipsoidal shape of the investigated star. Neglecting the gravity-darkening phenomenon leads to further overestimation of the magnetic dipole strength. The degree of this overestimation obtained from the mean crossover effect observations is most sensitive to the increase of the gravity-darkening exponent and to the character of the rotational law. The data of the mean longitudinal magnetic field provide the most correct estimation of the magnetic dipole strength value in the ellipsoidal star.
\end{abstract}

Key words. stars: chemically peculiar - stars: magnetic fields - stars: rotation

\section{Introduction}

Information about the effective temperature, chemical abundance, presence and variability of a magnetic field, etc. on a chemically peculiar (CP) star is usually obtained through the analysis of its spectra. The set of line profiles that forms a spectrum of the star is generated at different depths of the stellar atmosphere. The upper layer of stellar gas is stressed by the mutual action of radiation and gas pressure, centrifugal and gravitational forces and forms the resulting (often symmetrically spherical) shape of the stellar surface.

In order to describe this stellar surface shape mathematically, Liapunov (1903) considered a star as an isolated selfgravitational celestial body with rigid rotation and a barotropic fluid. According to him, the surfaces of equal density, pressure and potential of gravitational and centrifugal forces coincide and form an isobaric shell that can be represented by a Maclaurin ellipsoid. The ellipsoid's equation depends on the parameter $q$ which to a first approximation is equal to the ratio of centrifugal to gravitational forces at the equator (Liapunov 1903) and specifies the eccentricity of the ellipsoid (Kholshevnikov \& Elkin 2002). Moreover, Zaky et al. (1994) showed recently that the isobaric shell can be represented by axisymmetrical figures only. In such a way, for a slowly rotating star the centrifugal forces are comparatively small and not can sufficiently deform the spherical stellar shape. The eccentricity of the modelled Maclaurin ellipsoid is close to unity, and a star has an almost spherically symmetric form. But for a rapidly rotating star such a deformation could lead to observable stellar oblation at the rotational poles (van Belle et al. 2001).

High stellar rotation leads to the surface brightness distribution, as was originally shown by von Zeipel (1924). The next models of a rotating star explored the impact of rotation upon both stellar effective temperature (Slettebak 1949) and stellar shape (Collins 1963, 1965). Generalization of the von Zeipel theorem to account for differential rotation was performed recently by Maeder (1999). Currently, a common model of a differentially rotating star is characterized by highly anisotropic turbulence which has a strong horizontal component (Zahn 1992; Maeder 2003). This horizontal turbulence strongly reduces the horizontal differential rotation, so that rotation varies only radially. This means that the angular velocity is uniform at the surface of isobaric shells and, consequently, the rotation is usually said to be "shellular". The presence of a magnetic field generated, for example, by the Tayler-Spruit dynamo mechanism does not affect the shape of the equipotentials, but strongly amplifies the horizontal coupling (Maeder \& Meynet 2003).

The structure of a surface magnetic field for a rigidly rotating spherically symmetric CP star can be sufficiently well described in the framework of the oblique rotator model with a dipole plus non-linear quadrupole field (Bagnulo et al. 1996) or in the framework of the magnetic charge description (MCD) method (Gerth et al. 1997; Khalack et al. 2003). For a differentially rotating $\mathrm{CP}$ star a simulation of the crossover effect data (surface magnetic field characteristic) is considered in Khalack (2002). All the models mentioned above do not take into 


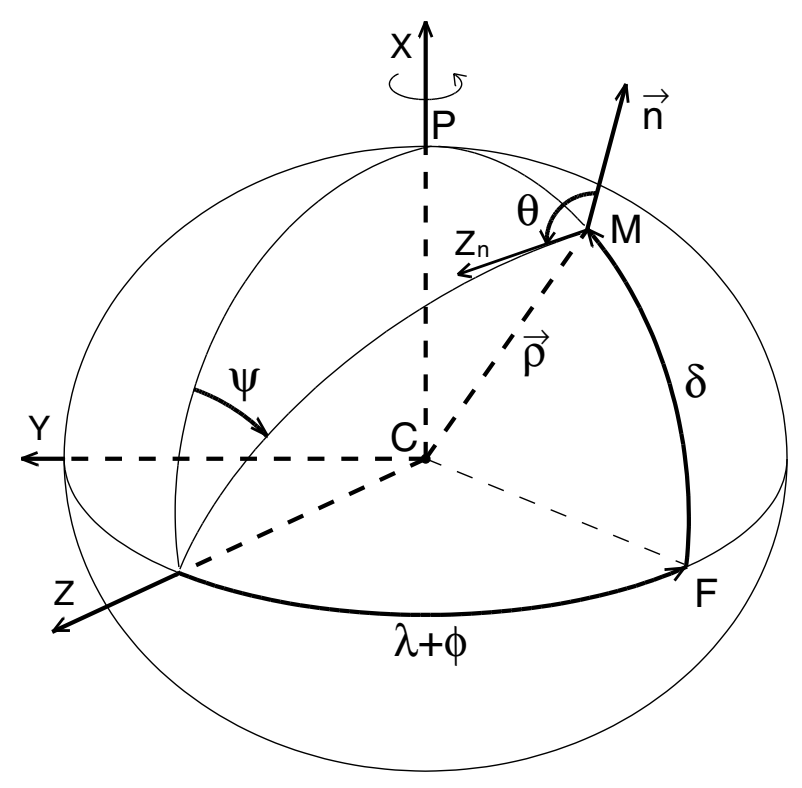

Fig. 1. Geometry of an ellipsoidal star.

account the effect of stellar oblation. The purpose of this paper is to consider stellar oblation influences on the results of surface magnetic field modelling. Section 2 specifies a dipole magnetic field vector at the surface of the Maclaurin ellipsoid in the framework of modified MCD approach (Khalack et al. 2001). Section 3 applies this magnetic field specification to the simplified case of a centered symmetric magnetic dipole and compares the results with the data obtained for a spherically symmetric star. The influence of the gravity-darkening phenomenon on the obtained results is discussed in Sect. 4. Section 5 presents the conclusions.

\section{Field of the magnetic dipole at the surface of a rotating ellipsoidal star}

\subsection{The main relations}

The surface of a rapidly rotating star has a shape that can be well described by the model of a rotating Maclaurin ellipsoid (Liapunov 1903; Collins 1963) in which a small semi-axis $R_{\mathrm{p}}$ is directed along the rotational axis, while a large semi-axis $R_{\mathrm{e}}$ lies in the equatorial plane. For the sake of convenience let us introduce the Spherical reference frame related to the Star (SS) with the origin in the stellar centre. For such a model the location of an arbitrary surface point $\mathrm{M}$ in the SS frame is specified by its latitude $\delta$ and longitude $\lambda$ (see Fig. 1), while its distance from the stellar centre in the $R_{\mathrm{p}}$ units is:

$\rho=\frac{1}{\sqrt{1-\varepsilon^{2} \cos ^{2} \delta}}$,

where eccentricity is defined as

$\varepsilon=\sqrt{1-\frac{R_{\mathrm{p}}^{2}}{R_{\mathrm{e}}^{2}}}$

Let us introduce also the Cartesian right handed reference frame related to the Observer $(\mathrm{CO})$ with the origin at the stellar centre and axes $Z$ directed to the observer and $X$ directed along the axis of rotation (see Fig. 1). If $\varphi$ is a phase of stellar rotation the aforementioned surface point $\mathrm{M}$ will have the $\mathrm{CO}$ frame coordinates that are linked to its coordinates in the SS frame by the following equations:

$\left\{\begin{array}{l}X=\rho R_{\mathrm{p}} \sin \delta \\ Y=-\rho R_{\mathrm{p}} \cos \delta \sin (\lambda+\varphi), \\ Z=\rho R_{\mathrm{p}} \cos \delta \cos (\lambda+\varphi) .\end{array}\right.$

Moreover, we can also consider the Spherical reference frame $(\theta, \psi)$ linked with the Observer (SO), where the variable $\theta$ specifies an angle between the line of sight and the normal to the ellipsoid's surface at the point $\mathrm{M}$, and the variable $\psi$ defines the positional angle of this point with respect to the ZOX plane. It is evident that in this situation the vector $\boldsymbol{\rho}$ and normal $\boldsymbol{n}$ are not co-linear (see Fig. 1). From the equation for a normal run to the point $\mathrm{M}$ at the ellipsoid's surface, we can deduce the following relations between the coordinates of this point in the SS and SO frames:

$\left\{\begin{array}{l}\sin \delta=\frac{X_{n}\left(1-\varepsilon^{2}\right)}{\sqrt{1-\varepsilon^{2} X_{n}^{2}\left(2-\varepsilon^{2}\right)}}, \\ \cos \delta=\frac{h}{\sqrt{1-\varepsilon^{2} X_{n}^{2}\left(2-\varepsilon^{2}\right)}} ;\end{array}\right.$

and

$\left\{\begin{array}{l}\sin \lambda=-Y_{n} / h \\ \cos \lambda=Z_{n} / h\end{array}\right.$

where $h=\sqrt{Y_{n}^{2}+Z_{n}^{2}}$ and

$\left\{\begin{array}{l}X_{n}=\cos \theta \cos i+\sin \theta \sin i \cos (\psi-\Omega), \\ Y_{n}=-\sin \theta \sin (\psi-\Omega), \\ Z_{n}=\cos \theta \sin i-\sin \theta \cos i \cos (\psi-\Omega) .\end{array}\right.$

Here the angle $i$ specifies the inclination of the stellar rotational axis to the line of sight while the variable $\Omega$ defines its positional angle.

\subsection{Integration over the visual stellar surface}

For the rotating ellipsoid (with $R_{\mathrm{p}}=1$ ) an elemental surface square is defined in the SS frame as

$\mathrm{d} S=\rho \sqrt{\rho^{2}+\left(\frac{\partial \rho}{\partial \delta}\right)^{2}} \cos \delta \mathrm{d} \delta \mathrm{d} \lambda$,

where $\rho$ is specified in Eq. (1). Based on Eqs. (4), (5) we can calculate the converting Jacobian and also define this elemental surface square in the SO frame as

$\mathrm{d} S=\frac{\sin \theta}{\left(1-\varepsilon^{2} X_{n}^{2}\right)^{2}} \mathrm{~d} \theta \mathrm{d} \psi$.

Imposing the limb darkening in the form $1-u+u \cos \theta$ we obtain the following expression for the integrated radiation flux from the visual surface of a rotating ellipsoid:

$I_{0}=\oint \mathrm{d} S \cos \theta(1-u+u \cos \theta)\left(1-\varepsilon^{2} \cos ^{2} \delta\right)^{\gamma}$. 
For a star with a weak oblation Eq. (9) can be expanded into a series in the small parameter $\varepsilon^{2}$

$I_{0} \simeq \frac{\pi}{30}\left[10(3-u)+\varepsilon^{2}\left(15\left(1+\cos ^{2} i\right)-u\left(7-\cos ^{2} i\right)\right)\right]$,

where only the first two terms are taken into account. Neglect of the rest of the terms leads to an error up to $0.012 \%$ from the result given by Eq. (10).

\subsection{Components of the surface magnetic field vector}

In the MCD method a surface magnetic field structure is described by the model of a magnetic dipole, mathematically formulated with the help of two virtual magnetic charges arbitrarily located in the stellar interior (Khalack et al. 2001). The detailed description of this model is given in Khalack et al. (2003) for the case of a spherically symmetric star.

Let us suppose that the $j$ th virtual magnetic charge $(j=$ $1,2)$ is located on the centered sphere of a radius $a_{j} R_{\mathrm{p}}$ in the stellar interior and has the coordinates $\theta_{j}, \psi_{j}$ in the SO frame. Then the distance between the $j$ th magnetic charge and the surface point $\mathbf{M}(\theta, \psi)$ is specified as

$r_{j}=R_{\mathrm{p}} \sqrt{\rho^{2}+a_{j}^{2}-2 a_{j} \rho D_{j}}$,

where

$D_{j}=\sin \theta \sin \theta_{j} \cos \left(\psi-\psi_{j}\right)+\cos \theta \cos \theta_{j}$

and $\rho$ is described by Eq. (1) and in the SO frame it has the form

$\rho=\sqrt{\frac{1-\varepsilon^{2} X_{n}^{2}\left(2-\varepsilon^{2}\right)}{\left(1-\varepsilon^{2}\right)\left(1-\varepsilon^{2} X_{n}^{2}\right)}}$.

The expressions for the Cartesian components of the magnetic field vector at the point $\mathrm{M}(\theta, \psi)$ of ellipsoid's surface can be deduced from Khalack et al. (2001) as

$$
\left\{\begin{aligned}
B_{x}=\frac{Q_{r}}{R_{\mathrm{p}}^{2}} & {\left[\frac{\rho \sin \theta \cos \psi-a_{1} \sin \theta_{1} \cos \psi_{1}}{\left(\rho^{2}+a_{1}^{2}-2 a_{1} \rho D_{1}\right)^{3 / 2}}\right.} \\
& \left.-\frac{\rho \sin \theta \cos \psi-a_{2} \sin \theta_{2} \cos \psi_{2}}{\left(\rho^{2}+a_{2}^{2}-2 a_{2} \rho D_{2}\right)^{3 / 2}}\right] ; \\
B_{y}=-\frac{Q_{r}}{R_{\mathrm{p}}^{2}} & {\left[\frac{\rho \sin \theta \sin \psi-a_{1} \sin \theta_{1} \sin \psi_{1}}{\left(\rho^{2}+a_{1}^{2}-2 a_{1} \rho D_{1}\right)^{3 / 2}}\right.} \\
& \left.-\frac{\rho \sin \theta \sin \psi-a_{2} \sin \theta_{2} \sin \psi_{2}}{\left(\rho^{2}+a_{2}^{2}-2 a_{2} \rho D_{2}\right)^{3 / 2}}\right] ; \\
B_{z}=\quad \frac{Q_{r}}{R_{\mathrm{p}}^{2}} & {\left[\frac{\rho \cos \theta-a_{1} \cos \theta_{1}}{\left(\rho^{2}+a_{1}^{2}-2 a_{1} \rho D_{1}\right)^{3 / 2}}\right.} \\
& \left.-\frac{\rho \cos \theta-a_{2} \cos \theta_{2}}{\left(\rho^{2}+a_{2}^{2}-2 a_{2} \rho D_{2}\right)^{3 / 2}}\right]
\end{aligned}\right.
$$

Here the variables $Q_{\mathrm{r}}$ and $a_{1}, a_{2}$ specify the modulus of the magnetic charges $\left(Q_{\mathrm{r}}=Q_{1}=-Q_{2}\right)$ and their distances from the centre of the star, expressed in the units of the ellipsoid's small semi-axis $R_{\mathrm{p}}$ (see Fig. 1), while $D_{1}$ and $D_{2}$ are described in Eq. (12). The variables $\left(\theta_{1}, \psi_{1}\right)$ and $\left(\theta_{2}, \psi_{2}\right)$ determine the angular coordinates of two magnetic charges in the SO frame.
Respective to Eq. (14), the mean (averaged over the visual ellipsoid's surface) longitudinal magnetic field can be calculated as

$\left\langle B_{z}\right\rangle=\frac{1}{I_{0}} \oint \mathrm{d} S B_{z} \cos \theta(1-u+u \cos \theta)$.

\section{Centered symmetric magnetic dipole}

For the centered symmetric magnetic dipole the coordinates of the two virtual magnetic charges in Eqs. (11), (12) are linked by the following relations: $a_{1}=a_{2}=a \ll 1, \delta_{1}=-\delta_{2}$ and $\lambda_{1}=\lambda_{2}+\pi$. Then, according to Eqs. (4), (5) $\cos \theta_{1}=-\cos \theta_{2}$, $\psi_{1}=\psi_{2}+n \pi$, where $n=0,1$ and $D_{1}=-D_{2}$. Also, we can introduce the angle between the magnetic dipole axis and the axis of axial stellar rotation as $\beta=\frac{\pi}{2}-\delta_{1}$.

\subsection{The mean longitudinal magnetic field}

Under the conditions mentioned above, Eq. (14) can be expanded into a series in the small parameter $a$ :

$\left\{\begin{array}{l}B_{x} \simeq \frac{2 a Q}{R_{\mathrm{p}}^{2} \rho^{3}}\left(3 D_{c} \sin \theta \cos \psi-X_{0} \cos \Omega+Y_{0} \sin \Omega\right), \\ B_{y} \simeq-\frac{2 a Q}{R_{\mathrm{p}}^{2} \rho^{3}}\left(3 D_{c} \sin \theta \sin \psi-Y_{0} \cos \Omega-X_{0} \sin \Omega\right), \\ B_{z} \simeq \frac{2 a Q}{R_{\mathrm{p}}^{2} \rho^{3}}\left(3 D_{c} \cos \theta-Z_{0}\right),\end{array}\right.$

where only the first term is taken into account, while

$D_{c}=\left(X_{0} \cos (\psi-\Omega)+Y_{0} \sin (\psi-\Omega)\right) \sin \theta+Z_{0} \cos \theta$,

$\left\{\begin{array}{l}X_{0}=\rho_{1}\left(\cos \beta \sin i-\left(1-\varepsilon^{2}\right) \sin \beta \cos i \cos \left(\lambda_{1}+\varphi\right)\right), \\ Y_{0}=\rho_{1}\left(1-\varepsilon^{2}\right) \sin \beta \sin \left(\lambda_{1}+\varphi\right) \\ Z_{0}=\rho_{1}\left(\cos \beta \cos i+\left(1-\varepsilon^{2}\right) \sin \beta \sin i \cos \left(\lambda_{1}+\varphi\right)\right)\end{array}\right.$

and

$\rho_{1}=\frac{1}{\sqrt{\left(1-\varepsilon^{2}\right)^{2} \sin ^{2} \beta+\cos ^{2} \beta}}$.

As the next step, we can expand the $B_{z}$ component from Eq. (16) into a series in the small parameter $\varepsilon^{2}$ and integrate it over the visual ellipsoid's surface according to Eq. (15). This simplification leads to the following expression for the mean longitudinal magnetic field

$$
\begin{aligned}
\left\langle B_{z}\right\rangle \simeq & \frac{\pi Q a}{30 I_{0} R_{\mathrm{p}}^{2}}\left\{2 Z_{0}(15+u)+3 \varepsilon^{2} X_{0} \sin i \cos i(35-11 u)\right. \\
& \left.-\varepsilon^{2} Z_{0}\left(15\left(3-7 \cos ^{2} i\right)-u\left(5-17 \cos ^{2} i\right)\right)\right\},
\end{aligned}
$$

where only the two terms are taken into account. Neglect of the rest of the terms leads to a maximum error of about $0.02 \%$ in $\left\langle B_{z}\right\rangle$ estimation for $i=0^{\circ}, \varepsilon=0.1, u=0.6$.

Equation (19) has a term proportional to $X_{0}$, which arises due to the fact that variables $\mathrm{d} S$ and $\rho$ are functions of $\cos (\psi-\Omega)$ (see Eqs. (6), (8) and (13)). After the expansion of Eq. (16) into a series in the parameter $\varepsilon^{2}$, we still have the 


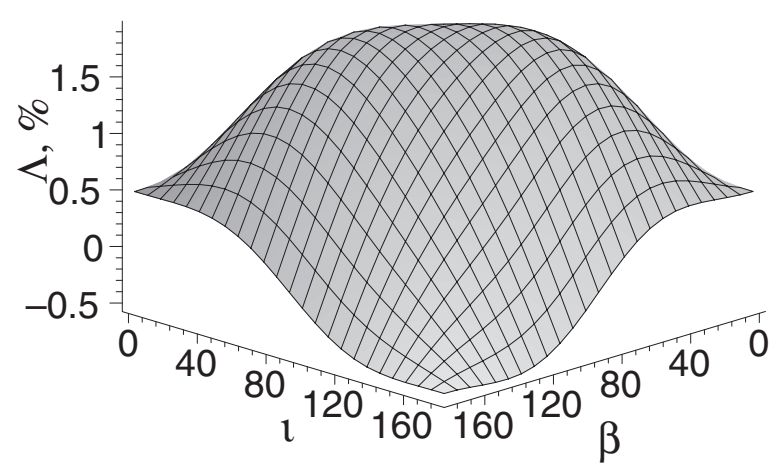

Fig. 2. The shape of the $\Lambda$-function for the mean longitudinal magnetic field at the values of $\varepsilon=0.3, u=0.6$ and $\varphi=0$.

parts proportional to $\cos ^{2}(\psi-\Omega)$ which not can be cancelled by the procedure of integration on $d \psi$ in Eq. (15).

In order to estimate the contribution of the stellar oblation effect, we calculate a ratio

$\Lambda(\varepsilon, u, i, \beta, \varphi)=\frac{\left\langle B_{z}(0)\right\rangle-\left\langle B_{z}(\varepsilon)\right\rangle}{\left\langle B_{z}(0)\right\rangle_{\max }} \times 100 \%$,

where $\left\langle B_{z}(\varepsilon)\right\rangle$ denotes the mean longitudinal magnetic field estimation for the case of nonzero stellar oblation, while $\left\langle B_{z}(0)\right\rangle$ is the field estimation obtained by neglecting the stellar oblation effect $(\varepsilon=0)$. For certain sets of the variables $i, \beta$ and $\lambda_{1}$ the mean longitudinal magnetic field estimation $\left\langle B_{z}(0)\right\rangle=0$. Therefore, in order to avoid a division by zero, we use in the denominator of Eq. (20) the maximum value of the mean longitudinal magnetic field $\left\langle B_{z}(0)\right\rangle_{\max }$ (when $i=0^{\circ}$ or $\beta=0^{\circ}$ ) for the spherically symmetric star. In Fig. 2 this ratio is plotted as a function of inclination angle $i$ and angle $\beta$ for the constant values of $\varepsilon=0.3, u=0.6$ and $\varphi=0$. The ratio depends on the value of the limb darkening coefficient, that leads to the weak increase of the $\Lambda$-function with the decrease of $u$. There is also the dependence of $\Lambda$-function on the phase $\varphi$ of stellar rotation (see Fig. 3). It originates from the fact that the orientation of the magnetic dipole axis in the $\mathrm{CO}$ frame (see Eq. (17)) depends on the eccentricity of the ellipsoidal star. The longitudinal component of the surface magnetic field in this case varies in a more sophisticated way with the stellar rotation than for the spherically symmetric star.

Nevertheless, as it follows from Figs. 2 and 3 the contribution of the stellar oblation effect to the estimation of $\left\langle B_{z}\right\rangle$ does not exceed $2 \%$ (or $\sim 2.5 \%$ for small $u$ ).

\subsection{Crossover effect}

The observable value of the crossover effect depends on a combined contribution of the Zeeman and Doppler effects into a line profile asymmetry of the Stokes $\mathrm{V}$ parameter averaged over the visible stellar disk for a given rotation phase (Mathys 1995a). Therefore, we first need to describe the structure of the surface magnetic field together with the velocity law for stellar rotation.

To describe the velocity law we must first choose between the rigid and differential rotation of the CP star. As it follows from the result of numerical MHD simulations of thick stellar

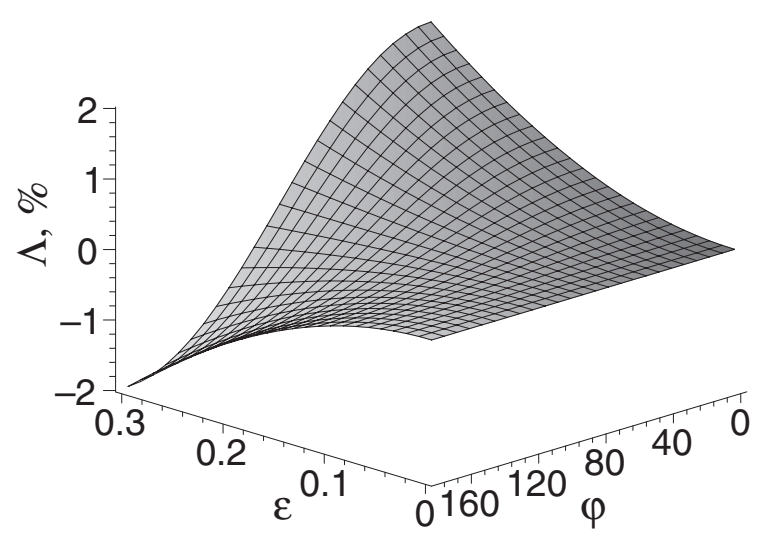

Fig. 3. The same as Fig. 2, but with the values of $u=0.6, i=90^{\circ}$ and $\beta=90^{\circ}$.

shells performed recently by Arlt et al. (2003), the time-scale of decay of differential rotation in the A stars is comparable with their life-time. On the other hand the strong differential rotation will evidently induce major distortion in the line profiles, which are not observed in the majority of CP stars (Mathys 2001). Nevertheless, we assume here a moderate differential rotation, which may be present in the $\mathrm{CP}$ stars during a considerable period of their life, in order to compare its influence to the magnetic dipole strength estimation with the impact of the stellar oblation effect.

Thus in this paper we are dealing with an ellipsoidal star that rotates with the solar differential rotation law (Gadun et al. 1985), which can be considered as a commonly used law of stellar differential rotation. The rotational velocity of the star depends on the stellar latitude $\delta$ (in the SS frame) as

$v(\delta)=v_{\mathrm{e}} \cos \delta\left(1-b \sin ^{2} \delta\right) \rho \sqrt{1-\varepsilon^{2}}$,

where $v_{\mathrm{e}}$ is the equatorial velocity and parameter $0<b \leq 1$, while the variables $\rho$ and $\varepsilon$ are specified by the Eqs. (1) and (2) respectively. For a rigidly rotating $\operatorname{star}(b=0)$, after the expansion of Eq. (21) into a series in the small parameter $\varepsilon^{2}$, we see that

$v(\delta) \simeq v_{\mathrm{e}} \cos \delta\left(1-\frac{\varepsilon^{2}}{2} \sin ^{2} \delta\right)$.

The form of Eq. (22) also corresponds to the solar differential rotation law for a spherically symmetric star (Khalack 2002), where parameter $b_{\mathrm{r}} \simeq \varepsilon^{2} / 2$.

A projection of the rotation velocity $v(\delta)$ from Eq. (21) onto the line of sight in the SO frame is $v_{\mathrm{z}}=-v(\delta) Y_{n} \sin i / \sqrt{1-X_{\mathrm{n}}^{2}}$, where the variables $Y_{n}$ and $X_{n}$ are determined in Eq. (6). According to Eq. (4) we can express the radial component of the rotation velocity through $X_{n}$ and $Y_{n}$ and then expand it into a series in $\varepsilon^{2}$. Finally, substituting the local longitudinal magnetic field $B_{z}$ in Eq. (15) with the local crossover effect $B_{\mathrm{c}}=B_{z} v_{\mathrm{z}}$, we can obtain the expression for the mean crossover effect in the differentially rotating ellipsoidal star

$$
\begin{aligned}
\left\langle B_{\mathrm{c}}\right\rangle \simeq & -\frac{\pi Q a v_{\mathrm{e}} Y_{0} \sin i}{560 I_{0} R_{\mathrm{p}}^{2}}\{56(8-3 u) \\
& -b\left[64\left(1+2 \cos ^{2} i\right)-u\left(29+23 \cos ^{2} i\right)\right] \\
& \left.-4 \varepsilon^{2}\left[8\left(13-16 \cos ^{2} i\right)-u\left(34-23 \cos ^{2} i\right)\right]\right\} .
\end{aligned}
$$




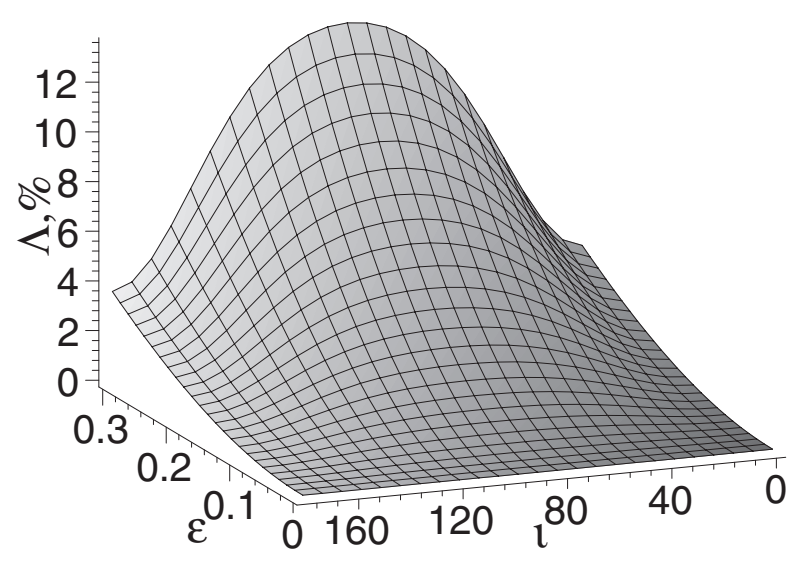

Fig. 4. The shape of the $\Lambda$-function for the mean crossover effect (see text) for $u=0.6$ and $b=0.2$.

Here only the first term and the terms linear on $b$ and $\varepsilon^{2}$ are taken into account and the variable $Y_{0}$ is specified by Eq. (17). Neglect of the rest of the terms leads to a maximum error of about $0.01 \%$ in $\left\langle B_{\mathrm{c}}\right\rangle$ for $i=0^{\circ}, \varepsilon=0.1, b=0.2$ and $u=0.6$.

The first two terms of Eq. (23) are in good agreement with the equation for the mean crossover effect in a spherically symmetric differentially rotating star (Khalack 2002). The third term reflects a contribution of the stellar oblation to the observed value of the mean crossover effect. The character of this correction can be verified through the ratio between the expressions for the mean crossover effect in the case of stellar oblation and without it:

$\Lambda(\varepsilon, u, i, \beta, \varphi)=\left(1-\frac{\left\langle B_{\mathrm{c}}(\varepsilon)\right\rangle}{\left\langle B_{\mathrm{c}}(0)\right\rangle}\right) \times 100 \%$.

In Fig. 4 we can see the dependence of the aforementioned ratio on the inclination angle $i$ and $\varepsilon$ for the constant value of $b=0.2$, which is close to the Solar value (Gadun et al. 1985), and $u=0.6$. This picture will not change essentially if we consider the case of solid stellar rotation, when $b=0$. The $\Lambda$-function does not depend on the angle $\beta$ and weakly depends on the parameter of stellar differential rotation $b$. Under $\varepsilon=0.3$ the effect of stellar oblation increases the theoretical $\left\langle B_{\mathrm{c}}\right\rangle$ value, calculated for the spherically symmetric star, by $6-12 \%$. In other words, if we neglect the effect of stellar oblation we will overestimate the strength of the magnetic dipole $B_{\mathrm{d}} \simeq 4 Q a / R_{\mathrm{p}}^{2}$, supposing that the equatorial velocity $v_{\mathrm{e}}$ of a star is well determined from the magnetically insensitive spectral lines.

\subsection{The mean quadratic magnetic field}

According to Mathys (1995b) the mean quadratic magnetic field is determined by $\left\langle B_{\mathrm{mq}}\right\rangle^{2}=\left\langle B^{2}\right\rangle+\left\langle B_{z}^{2}\right\rangle$. In the same way, for the central symmetric magnetic dipole we can expand the other surface magnetic field components (14) into a series in the small parameter $a$, as it was made for $B_{z}$ (see Eq. (16)), and calculate the expression for the mean quadratic magnetic field. Substituting the local longitudinal magnetic field $B_{z}$ in Eq. (15)

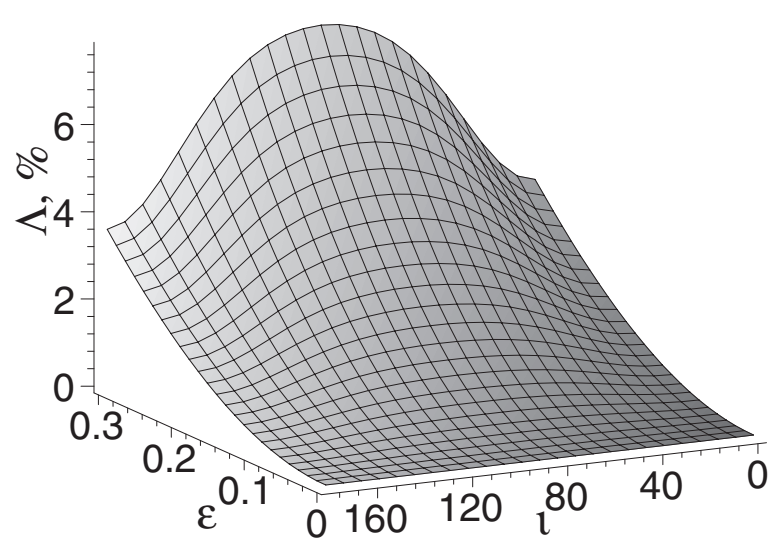

Fig. 5. The shape of the $\Lambda$-function for the mean quadratic magnetic field (see text) at $u=0.6$ and $\beta=90^{\circ}, \varphi=0$.

by this expression, we obtain:

$$
\begin{aligned}
\left\langle B_{m q}\right\rangle^{2} \simeq & \frac{4 \pi a^{2} Q^{2}}{I_{0} R_{\mathrm{p}}^{4}}\left\{\frac{525-193 u}{210}+Z_{0}^{2} \frac{105+13 u}{105}\right. \\
& +\varepsilon^{2} *\left(\frac{105-247 u}{140}+Z_{0} X_{0} \sin i \cos i(21-5 u)\right. \\
& +\sin ^{2} i\left[X_{0}^{2} \frac{245-117 u}{40}-\frac{2205-157 u}{240}\right] \\
& \left.\left.+Z_{0}^{2}\left[\frac{1365-181 u}{140}-\sin ^{2} i \frac{651-11 u}{48}\right]\right)\right\}
\end{aligned}
$$

The third term in Eq. (25) is responsible for the contribution of the stellar oblation to the observed value of the mean quadratic magnetic field. In order to estimate this contribution we analysed the behaviour of the $\Lambda(\varepsilon, u, i, \beta, \varphi)$-function that is specified by Eq. (24), in which the expression for $\left\langle B_{\mathrm{c}}(\varepsilon)\right\rangle$ was substituted by $\left\langle B_{\mathrm{mq}}(\varepsilon)\right\rangle$. In Fig. 5 we can see the dependence of the $\Lambda$-function calculated for the mean quadratic magnetic field on the inclination angle $i$ and $\varepsilon$ for the constant values of $u=0.6$ and $\beta=90^{\circ}, \varphi=0$. It weakly depends on the limb darkening coefficient $u$ and varies within $2 \%$ with the phase $\varphi$ of stellar rotation. Nevertheless, in Fig. 6 we can see the comparatively strong dependence of the $\Lambda$-function on the angle $\beta$. Figures 5 and 6 show that at $\varepsilon=0.3$ the theoretical value of $\left\langle B_{\mathrm{mq}}(\varepsilon)\right\rangle$ can exceed the $\left\langle B_{\mathrm{mq}}(0)\right\rangle$ up to $4-8 \%$. That could lead to the essential overestimation of the magnetic dipole strength obtained from the mean quadratic magnetic field observations if we did not pay attention to the ellipsoidal shape of the investigated star.

\section{Discussion}

For an ellipsoidal star, its polar zones distorted by rapid rotation are hotter than its equatorial zones, because $T_{\text {eff }}^{4} \sim\left(g_{\text {eff }}\right)^{\beta_{1}}$ (von Zeipel 1924) and the effective gravity is higher at the poles of the star. This phenomenon is known as gravity-darkening, although some authors prefer to call it gravity-brightening. The result obtained by von Zeipel (1924) with the gravity-darkening 


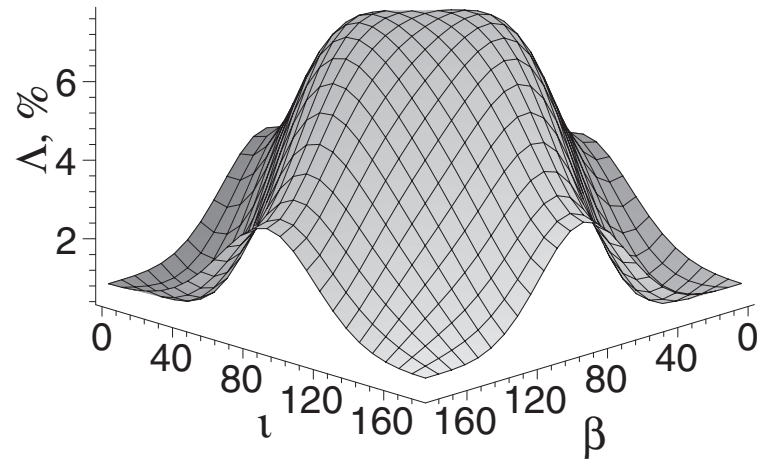

Fig. 6. The same as Fig. 5, but with $\varepsilon=0.3, u=0.6$ and $\varphi=0$.

exponent $\beta_{1}=1$ is valid for configurations in radiative equilibrium. The value of $\beta_{1}$ depends on the internal structure of the star. For the stars with conservative rotation laws and radiative envelopes Smith et al. (1974) obtained $\beta_{1}=0.5$, while Ludwig et al. (1999) indicated that for stars with convective envelopes the gravity-darkening exponent is around 0.28-0.40. Claret (2000) pointed out that the gravity-darkening phenomenon is related strongly to the details of the rotation law of the star. Furthermore, for a magnetic CP star the effective gravity depends also on the magnetic pressure and, consequently, on the surface magnetic field structure.

In the present paper, in order to account for the gravitydarkening phenomenon, we construct a simplified approach supposing that the effective gravity at the stellar surface is inversely proportional to the square of its distance from the center of the star. The ordinary limb darkening law is combined with the brightness distribution over the stellar surface $\rho^{-2 \gamma}$, which has a latitude dependence (see Eq. (1)). This expression is very approximate and the variable $0<\gamma<1$ is only related to $\beta_{1}$ and is not equivalent to it.

According to the new limb darkening law the new expressions for the integrated radiation flux as well as for the mean longitudinal magnetic field, the crossover effect and the quadratic field are obtained and the respective $\Lambda$-functions are recalculated. Choosing the values of the rest of the parameters in Eqs. (20), (24) that they provide the maximum of $\Lambda$-function (see Figs. 2, 4 and 6), we analyse its dependence on the new parameter $\gamma$. Figure 7 shows that for all the surface magnetic field characteristics their $\Lambda$-function grows with the increase of the $\gamma$ parameter. The ordinary case with $\gamma=0$ was discussed in Sect. 3 and should be considered as a case that gives us the lower limit to the possible overestimation of the magnetic dipole strength from observations if we ignore the effect of stellar oblation. By increasing the value of the parameter $\gamma$ we can change the brightness distribution over the surface of the star and, consequently, the difference of $T_{\text {eff }}$ values at the stellar pole and equator. As follows from Fig. 7 , the $\Lambda$-function obtained for the mean longitudinal magnetic field is almost insensitive to the increase of $\gamma$, while for the mean crossover effect the $\Lambda$-function increases to $2 \%$, when we increase the parameter $\gamma$ from 0 to 1 . It should be stressed here that this dependence has only a qualitative character and should be considered as a first approximation, especially for the mean crossover

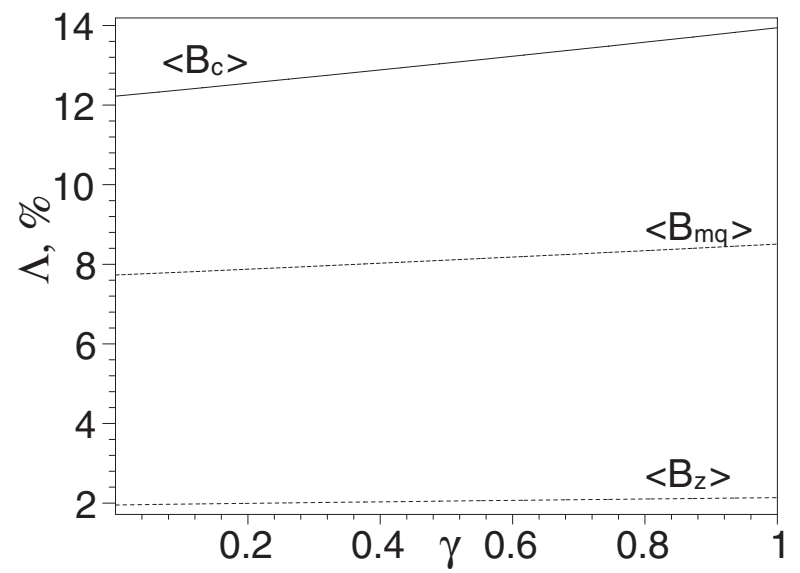

Fig. 7. Dependence of the $\Lambda$-function on the gravity-darkening parameter for the mean longitudinal magnetic field (dotted line), crossover effect (solid line) and quadratic field (dashed line).

effect, because the gravity-darkening phenomenon is strongly related to the character of stellar rotation (Claret 2000). In the accepted law of the stellar brightness distribution we did not take into account the possibility of differential rotation as well as the impact of the surface magnetic field structure.

In Eq. (23) we suppose that the equatorial velocity $v_{\mathrm{e}}$ of the star is well determined from the magnetically insensitive spectral lines. Its value can be estimated from the analysis of unpolarized line profiles, which also depend on the geometrical configuration of the stellar surface (Collins 1963, 1965). In general, for an ellipsoidal star the measured value of $v_{\mathrm{e}}$ is usually underestimated due to the complex character of the rotational law, that leads to further overestimation of the magnetic dipole strength $B_{\mathrm{d}} \simeq 4 Q a / R_{\mathrm{p}}^{2}$, if we neglect the effect of stellar oblation.

The observable values of the surface magnetic field characteristics discussed above were obtained from the line profile analysis of the magnetically sensitive spectral lines in polarized stellar spectra (Mathys 1991, 1995a,b; Mathys \& Hubrig 1997) supposing the rigidly rotating and spherically symmetric model for the investigated stars. But for a star with essential axial rotation the local flux depends on the gravity according to von Zeipel's theorem and a resulting polarized line profile can differ from the line profile that is usual for a spherically symmetric star (Harrington \& Collins 1968). This means that correction of the magnetic field measurements for the stellar oblation effect and the corresponding gravity-darkening should be performed at the stage of line profile analysis or later, during the modeling of stellar magnetic field structure.

\section{Summary}

Currently, most of the known CP stars are characterized by their strong surface magnetic field (Romanyuk 1997; Bychkov et al. 2003). Some of them have slow axial rotation, while others show sufficiently high equatorial rotational velocity (Catalano \& Renson 1998; Hubrig et al. 2000) to argue for the deformation of the stellar surface. It is widely accepted 
to consider a spherically symmetric star in the existing models of the magnetic field structure at the stellar surface. An estimate of the strength of the magnetic dipole (in the model of an oblique rotator with the dipole magnetic field) based on the main surface magnetic field characteristics such as the mean longitudinal magnetic field (Mathys 1991), crossover effect (Mathys 1995a) and quadratic field (Mathys 1995b) is a function of the geometrical characteristics of the stellar surface (Bagnulo et al. 1996; Khalack et al. 2001). In this situation the main question is: How strong should this deformation be in order to distort our estimation of the magnetic dipole strength?

This paper gives the approximate expressions for the theoretical estimation of the mean longitudinal magnetic field (see Eq. (19)), the mean crossover effect (see Eq. (23)) and the mean quadratic field (see Eq. (25)) in the framework of the ellipsoidal star model of an oblique rotator with a centred symmetric magnetic dipole.

From the analysis of Eqs. (19), (23), (25) it follows that the aforementioned magnetic field characteristics do not depend on the positional angle $\Omega$ and are not equally sensitive to the stellar oblation effect. For the mean longitudinal magnetic field we can ignore the contribution of the oblation effect because it does not exceed 2\% (see Figs. 2 and 3). Nevertheless, for the mean crossover effect and the mean quadratic field the effect of stellar oblation increases their theoretical value (under $\varepsilon=0.3$ ) up to $12 \%$ (see Fig. 4) and up to $8 \%$ (see Figs. 5 and 6) respectively. That could lead to the essential overestimation of the magnetic dipole strength value obtained from the mean crossover effect and quadratic magnetic field observations if we neglect the effect of stellar oblation.

Based on Eq. (14) we can calculate the magnetic field characteristics for an ellipsoidal star with a complex surface magnetic field structure. They also do not depend on the positional angle and without a loss of generality, we can set $\Omega=0^{\circ}$ in these calculations.

Acknowledgements. The author is grateful to the referee for his/her constructive remarks and recommendations and to Ms. J. Anderson and Ms. N. Schneider-Larby for their valuable advice and help with text revision.

\section{References}

Arlt, R., Hollerbach, R., \& Rüdiger, G. 2003, A\&A, 401, 1087

Bagnulo, S., Landolfi, M., \& Landi Degl'Innocenti, M. 1996, A\&A, 308, 115

Bychkov, V. D., Bychkova, L. V., \& Madej, J. 2003, A\&A, 407, 631

Catalano, F. A., \& Renson, P. 1998, A\&AS, 127, 421

Claret, A. 2000, A\&A, 359, 289

Collins II, G. W. 1963, ApJ, 138, 1134

Collins II, G. W. 1965, ApJ, 142, 265

Gadun, A. S., Kostyk, R. I., \& Sheminova, V. A. 1985, Kin. \& Phys. Cel. Bodies, 15, N6, 53

Gerth, E., Glagolevskij, Yu. V., \& Sholz, G. 1997, in Stellar Magnetic Fields, ed. Yu. V. Glagolevskij, \& I. I. Romanyuk (Moscow), 67

Harrington, P. J., \& Collins II, G. W. 1968, ApJ, 151, 1051

Hubrig, S., North, P., \& Mathys, G. 2000, ApJ, 539, 352

Khalack, V. R., Khalack, Yu. N., Shavrina, A. V., et al. 2001a, AZh, $78, \mathrm{~N} 7,655$

Khalack, V. 2002, A\&A, 385, 986

Khalack, V., Zverko, J., \& Žižňovský, J. 2003, A\&A, 403, 179

Kholshevnikov, K. V., \& Elkin, A. V. 2002, Celest. Mech. Dynam. Astron., 84, 57

Liapunoff, A. M. 1903, Notes de l'Académie Impériale des Sciences, 14,1

Ludwig, H.-G., Freitag, B., \& Steffen, M. 1999, A\&A, 346, 111

Maeder, A. 2003, A\&A, 347, 185

Maeder, A. 2003, A\&A, 399, 263

Maeder, A., \& Meynet, G. 2003, A\&A, 411, 543

Mathys, G. 1991, A\&AS, 89, 121

Mathys, G. 1995a, A\&A, 293, 733

Mathys, G. 1995b, A\&A, 293, 746

Mathys, G. 2001, private communication

Mathys, G., \& Hubrig, S. 1997, A\&AS, 124, 475

Romanyuk, I. I. 1997, The Zeeman effect in stellar spectra. In Proc. of International Conference, Stellar Magnetic Fields, ed. Y. V. Glagolevskij, \& I. I. Romanyuk (Moscow: Special Astrophys. Observ. Press), 11

Slettebak, A. 1949, ApJ, 110, 498

Smith, R. C., Smith, J. B., \& Worley, R. 1974, MNRAS, 167, 199

van Belle, G. T., Ciardi, D. R., Thompson, R. R., Akeson, R. L., \& Lada, E. A. 2001, ApJ, 559, 1155

von Zeipel, H. 1924, MNRAS, 84, 665

Zahn, J. P. 1992, A\&A, 265, 115

Zaky, S. F., Elkin, A. V., \& Kholchevnikov, K. V. 1994, AZh, 71, N5, 785 